\title{
Das Insectensieb,
}

\section{dessen Bedeutung beim Fange von Insecten, insbesondere Coleo- pteren und dessen Anwendung.}

Besprochen von Edm. Reitter in Mödling.

(Schluss.)

Das Laub der Eichenwälder pflegt besondere Arten zu beherbergen, jedoch auch das unscheinbare Laub der Coniferen birgt an günstigen, feuchten Localitäten eine Menge ausgezeichneter Kleinkäfer. Die sonnig gelegenen Auen in der Nähe der Bäche und Flüsse und das Moos geschützter Waldwiesen sind im ersten Frühjahre ein vortreffliches Siebeterrain. $\mathrm{Zu}$ derselben Zeit kann man in gut situirten Hainen oder unter vereinzelten freistehenden Baumgruppen, oder unter niederen dichten Gebüschen, welche oftmals die Grenze zweier Felder markiren, oder unter dem Gebüsche an sonnig gelegenen Gartenmauern, oder in den Gärten selbst mit dem Siebe gute Ausbeute von solchen Arten machen, die man unter dem Laube des Waldes und in Bergschluchten vermissen wird. Ebenso kann man im ersten Frühjahre die feuchtwarmen Strohabfälle bei Mistbeeten durch's Sieb schütteln; man wird daselbst gewöhnlich Staphyliniden, Euplecten, Acriten etc. vorfinden. Aus dem Laube, zwischen welchem sich Ameisen befinden, wird man Myrmedonien, Homeusen, und wenn man Glück hat, auch Euryusen herausfinden. Man versäume nicht die grossen Nester der Formica rufa und anderer Ameisenarten im ersten Frühjahre aufzusuchen und einzelne Partien aus der Mitte ihres Baues hervorzuholen und durch einige rasche Handgriffe in's Si $\_$b zu schaffen. Ich habe für diese Operation stets ein Sieb verwendet, das nur eine geringe Maschenweite besass; dabei habe ich die Vorsicht beobachtet, zwischen den schüttelnden Bewegungen einige Pausen der Ruhe eintreten $\mathrm{zu}$ lassen, welche den beunruhigten flüchtenden Myrmecophilen gestatteten, durch die Oeffnungen des Siebes in den Siebesack zu entkommen. Ich glaube nicht, nöthig zu haben, die zahlreichen Coleopteren-Arten anführen zu sollen, welche man auf diese Weise in Menge mit dem Gesiebe heimbringt. Die Bemerkung dürfte vielleicht nicht überflüssig sein, beim Aussuchen desselben, welcherVorgang selbstverständlich der zahlreichen Ameisen wegen, entweder in einem Gartenhause oder im Freien geschieht, darauf zu achten, dass man die winzigen, gewöhnlich zahlreich auftretenden Ptilien nicht übersieht. 
Frühjahrs übersehwemmungen, namentlich aber, wenn solche $\mathrm{zu}$ vorgeschrittenerer Jahreszeit stattfinden, liefern, wie jeder Entomologe weiss, eine zahllose Menge, zum Theile ausgezeichneter Insecten, besonders Coleopteren, und wenn man nicht vorzieht, das Gemülle an den Flussufern in grosse Säcke zu schaffen, die direct nach Hause getragen werden, so wird man durch den Umfang und die Stärke des Siebes in der Lage sein, sämmtliche Reservesäckchen, die man mit sich führt, mit durchgesiebtem, gutem Gemülle bald anzufüllen. Wie ich schon früher angedeutet habe, empfiehlt es sich, mehrere Siebe zu besitzen, deren Maschenweiten verschiedene sind. Dadurch wird es möglich, bei Hause das eingesiebte Flussgemülle durch ein dichteres Sieb durchpassiren zu lassen, wodurch die zeitraubende Arbeit des Aussuchens wesentlich verringert wird. Die groben Rückstände werden in flüchtiger Weise nach grösseren Käfern durchgesucht, bevor sie fortgeworfen werden.

Bei der Hantirung mit mehreren Sieben hat man in sehr feuchten Localitäten Siebe mit grosser Maschenweite zu wählen. Man wird selbst in diesem Falle verhältnissmässig nur geringe, aber desto werthvollere Siebepartikelchen in den Siebesack bekommen. Das Gesiebe aus sehr feuchten Localitäten muss häufig in Reservesäckchen überschüttet werden, weil es sich sonst zu kleinen Kugeln zusammenballt, welche oft die zarteren Insecten in sich einschliessen. Solches, sehr feucht eingerafftes Gesiebe vermeide man auch sogleich durchzusuchen. Man lasse es vielmehr 1-2 Tage lang an einem passenden Orte trocknen und drücke sodann vor dem Aussuchen die zusammengeballten Theile auseinander. In den Karpathen, dem feuchtesten Gebirge Europas, muss man diese Vorsichten beim Sieben stets gebrauchen, zumal auch hier sich die besten Arten auf die feuchtesten Stellen zurückziehen und mit dem triefenden Laube in's Sieb gebracht werden müssen.

Bei grösseren Sammelreisen wird man Siebe von verschiedener Maschenweite gar nicht entbehren können. Namentlich im Süden Europas werden die Umstände, unter welchen man das Sieb zum Einsammeln von Coleopteren gebrauchen kann, so mannigfach, dass es sich empfiehlt, verschiedene Siebe in Vorbereitung zu besitzen. Baummulm, dürre Rasenstücke, Humustheile etc. wird man stets in ein dichtes Sieb gelangen lassen, um Zeit und Mühe zu sparen. Ein anderer Vortheil 
mehrerer Siebe ist auch der, dass man Führer, Träger oder sonstige Personen zum Einsieben mit verwenden kann, insoweit es die vorhandenen Siebe gestatten.

Das erste Frühjahr ist sowohl bei uns, wie im Süden Europas für die Verwendung des Siebes die ergiebigste Sammelzeit. Es gibt zwar Fälle, wo der Sommer-Anfang lohnender sich gestalten könnte, wie z. B. in den sogenannten Trichtern des Capella-Gebirges in Croatien. Die Capella weist keine Wasserläufe oder Bäche auf, auch bildet sie wenige oder nur geringe Thalbildungen und Bergeinschnitte; ihre Böschungen sind aber von zahlreichen tiefen, mulden- oder trichterförmigen Höhlungen besetzt, welch letztere wieder, wie der Gebirgszug ïberhaupt, mit gemischtem Walde bestockt und mit Pflanzen üppig bewachsen sind. Diese Höhlungen werden im Winter mehr als die übrige Fläche mit Schnee gefüllt und bilden für die Niederschläge ein natürliches Reservoir, wodurch ihr bedeutender Feuchtigkeitsgehalt im Sommer zu erklären ist. Aus diesem Grunde bilden diese Trichter ein ungemein ergiebiges Sammelterrain für das Sieb zum Ende des Frühjahres und im Beginne des Sommers. Wer sich für die Fauna dieses eigenthümlichen Bodenterrains, welches ich zweimal besucht habe, interessirt, wolle meinen diesbezüglichen Artikel in den Schriften der Zoologisch-Botanischen Gesellschaft in Wien, 1879, pag. 35 nachlesen.

I m vorgeschrittenen Frïhjahre wird das Aus. sieben von Laub in den Auen, in Gärten, unter schattigen Gebüschen längs den Gartenmauern und an ähnlichen Localitäten wegen der abnehmenden Feuchtigkeit unergiebig. Der rationelleSammler muss zu dieser Zeit stets, dem Feuchtigkeitsgrade des Laubes folgend, schattige Waldschluchten aufsuchen, wo man, wie z. B. in Croatien, das in den kleinen Wasserläufen angestaute Laub mit ausgezeichnetem Erfolge durch das Sieb passiren lassen kann.

$\mathrm{Zu}$ Beginn des Sommers beachte man die Composthaufen, welche in grösseren Gärten angelegt werden. Diese bestehen aus ausgejäteten Pflanzen, die auf einem Haufen der Fäulniss überliefert und mit einer dünnen Erdschichte überlegt werden. Diesen Composthaufen kommen jene Unkrauthaufen gleich, welche namentlich im Süden Europas an den Rändern der Weingärten sich vorfinden und welche eine grosse Menge von Käfern enthalten, die allerdings meist gewöhnlichen Arten, wie z. B. aus den Gattungen Oxytelus, Eumicrus, Tri- 
chopteryx, Cercyon, Hister u. s. w. angehören. In Dalmatien, auf den dalmatinischen Inseln, in Griechenland u. s. w. pflegt man den frischen Stallmist auf besonderen Plätzen oder an den Rändern der Weinfelder aufzustapeln. Diese Düngerhaufen enthalten merkwürdiger Weise ausser Rhyssemus, Plagiogonus und Oxyomus wenig Aphodien; dagegen sind Staphyliniden und besonders zahlreiche Histeriden und Cercyonen in grosser Anzahl vorhanden. An diesen Localitäten habe ich Dendrophilus punctatus und Coelostoma dalmatinum stets in grösserer Anzahl angetroffen.

$\mathrm{Zu}$ Beginn des Sommers und im Sommer beachte man ganz besonders die alten Baumstöcke, die gefällten Bäume oder andere verfaulte oder faulende Holzüberreste. An frischen Baumstöcken, namentlich der Buchen oder Eichen, wird man, wenn aus ihnen noch Saft ausgeschwitzt wird, zahlreiche Clavicornen vorfinden. Bevor man von diesen Stöcken die Rinde löst, untersuche man das Moos, welches oft dieselben dicht umgibt, indem man das Sieb an dem untersten Theile des Stockes anhält und die oberen Theile über dem Siebe mit einem Beile vom Moose entblösst. Aus dem, vom Baumsafte durchsickerten Moose kann man Sphaerites glabratus, Tachinus proximus, Quedien und zahlreiche andere Arten in's Sieb bekommen. Bei der Ablösung von faulender Baumrinde hat man stets das Sieb mit zu benützen, und zwar in der Weise, dass die Rindenstücke durch heftige Schläge über dem Siebe ausgeklopft werden und ausserdem ist die von der Rinde entblösste Stelle des Stockes oder Baumstammes derart zu behandeln, dass der Baummulm in vorsichtiger Weise mit in das Sieb gelangt. Die Erfolge dieser Sammelmethode sind gewöhnlich ganz überraschende. Es wäre vergebens, die Arten aufzuzählen, welche auf diese Art in das Sieb gelangen. Auf solche Weise habe ich in Czap, in Nord-Ungarn, eine unglaubliche Menge guter Coleopteren eingesammelt, darunter Ptinella biimpressa n. sp. in Anzahl, Euplectus filum und Spinolae in grosser Menge, die mir allerdings aus den Leinwandsäckchen, in welche ich das Gesiebe geschafft hatte, im Lauf der nächsten drei Tage zum grossen Theile wieder entkamen.

$\mathrm{Faulende} \mathrm{Holzstämme} \mathrm{sollen} \mathrm{in} \mathrm{vorsichtiger} \mathrm{Weise}$ untersucht werden. Enthalten sie einzelne Coleopteren, so wird man gewiss deren in grösserer Anzahl auffinden, wenn man die Holztheilchen über dem dichten Siebe zerbröckelt und tüchtig durchschüttelt. Solche Stämme, auch wenn sie noch nicht 
vollends abgestorben sind, pflegen zahlreiche Schwämme zu tragen, welche partienweise in's Sieb geschafft und über demselben zerkleinert, eine reiche und willkommene Ausbeute liefern werden. Auf diese Weise wird in Ungarn und Siebenbürgen ausser zahlreichen Cioiden der seltene Derodontus und die geschätzte Mycetoma gesammelt. Wenn man Ursache hat, mit der Sammelzeit zu sparen, so lege man die Pilze und Schwämmchen in Reservesäckchen ein (die ein rationeller Sammler unter allen Umständen in einigen Stücken bei sich tragen wird) und zerkleinere sie über dem Siebe in freier Zeit erst zu Hause.

Grosse harte Baumschwämme, die sich in alten, niederschlagreichen oder feuchten Wäldern in grosser Anzahl vorfinden, enthalten auf ihrer Unterseite zu günstiger Zeit Cryptophagus crenatus, Atomaria alpina, Enicmus brevicollis, Tritomen, Sacien, Corticarien und andere seltenere Coleopteren. Diese Thiere sind leicht mittelst eines heftigen Schlages in das untergehaltene Sieb zu bringen. Die Baumschwämme kann man in Reservesäcken nach Hause nehmen und daselbst mit Musse nach ihrem Inhalte, der meist aus Bolitophagus, Diaperis, Orchesia, Dorcatoma etc. besteht, durchsuchen. An verpilzten Baumstämmen befinden sich oftmals Agathidien, die man bei richtiger Anwendung des Siebes in grossen Mengen erbeuten kann. An der verpilzten Stirnfläche eines faulenden Baumstammes bei Raho in Nord-Ungarn sah ich einige Agathidium discoideum sitzen. Ich schlug mit einem kleinen Handbeile eine mehrere Zoll tiefe Schichte des faulenden Holzes in das untergehaltene Sieb ein und fand beim Aussuchen dieses Gesiebes mindestens fünf Agathidium-Arten in grosser Anzahl vor, darunter das schöne $A$. discoideum und plagiatum in einigen Hundert Exemplaren. Das Aushauen von wunden oder faulenden Stellen stehender Bäume, namentlich der Eichen, besonders wenn aus solchen der Baumsaft quillt, ist stets lohnend auszuführen und soll niemals übersehen werden. Pleganophorus bispinosus lebt in anbrüchigen Eichen, welche von Lasius brunneus bewohnt werden. Der Entdecker dieses merkwürdigen Thieres sammelte sie um Hermannstadt im ersten Frühjahre, indem er die am Fusse der Stämme befindlichen Moospolster abhob, zwischen welchen die Ameisen ihre Gänge gebaut hatten und falls sich Pleganophorus nicht 
vorfand, oder nach Aufnahme desselben, die Moospolster wieder an die Stämme drückte.

Meine Absicht, das Thier an denselben Eichenstämmen im Juni 1876 auf gleiche Weise zu finden, misslang, weil die Zeit bereits eine zu vorgeschrittene war; selbst das Durchsieben des trockenen Baummooses, das von Ptinus subpilosus zahlreich belebt war, lieferte nicht das gewünschte Resultat. Erst das kräftige Losschlagen der knorrigen alten, theilweise losen Rindentheile, welche Operation natürlich über dem Siebe geschah, brachte mich in den Besitz eines weiblichen Pleganophorus, welcher der letzte war, der an dieser Localität überhaupt gefangen wurde. Auf ganz ähnliche Weise ist es mir gelungen, bei Triest Mitte April 1880 von alten Eichen die neue Triplax tergestana in einigen Stücken in's Sieb zu schlagen, die ich sonst nicht entdeckt haben würde. Durch das Angeführte soll nachgewiesen werden, dass es für den rationellen Sammler unlohnend ist, an Bäumen, liegenden Stämmen oder an Baumstöcken ohne Sieb mit dem Stemmeisen herum zu krabbeln, und die sich zeigenden Thiere sogleich in die Fangflaschen zu bringen. In dieser Weise wird nur ein sehr kleiner Bruchtheil dessen dem Sammler zur Beute, was er bei geringerem Zeitaufwande mit Benützung des Siebes hätte erlangen können.

Holzspäne, die oftmals in Wäldern in alten Holzschlägen oder an solchen Plätzen faulen, auf denen einmal Schindel erzeugt wurden, soll man stets der Beachtung werth finden und über dem Siebe durch heftige Schläge von ihren Erdtheilen entblössen. In diesem Gesiebe wird man oft Anommatus, Trichophya, Homalota etc. in Anzahl vorfinden. Selbst der Mist, welcher sich unter solchen Bäumen oftmals an Waldesrändern vorfindet, worauf die Haushühner sich ïber Nacht aufzubäumen pflegen, liefert durch das Einsieben gute Aleochara, Microglossa und andere Staphylinus-Arten.

Hat man unter Steinen solche A meisennester entdeckt, in denen sich Claviger, Hetaerius oder Catopomorphus befinden, so vermeide man es, nach den einzelnen Myrmecophilen zu haschen. Hat man das Sieb bei sich und der Siebesack ist leer, so scharre man möglichst rasch, bevor sich die Thiere in ihre tieferen Gänge flüchten können, das ganze Nest in's Sieb. Kann man im Momente das Sieb hierzu nicht benutzen, so genügt auch ein Reservesäckchen, aus dem man zu 
Hause durch's dichte Sieb die gröberen Erdtheile, Steinchen und Wurzelwerk entfernen kann. In ähnlicher Weise hat man zu verfahren, wenn man die Dermestes, Saprinus, Catops, Corynetes etc. aus A es ern hervorholen will. Wie unangenehm und lästig ist es, mit der Pincette die einzelnen Thiere aus den Cadavern herauszuholen und wie wenig wird die dabei in keineswegs angenehmer Weise verbrachte Zeit belohnt. Man suche das Aas rasch auf das weitmaschige Sieb zu legen und scharre möglichst schnell die Erde, worauf es vorher lag, mit den zahllosen Insecten, die sich in sie flüchten, mit einem Beile oder einem anderen Instrumente, das man eben bei der Hand hat, entweder in ein zweites Sieb oder in ein Reservesäckchen. Das Cadaver wird einige Male über dem Siebe ge-wendet und falls daran keine eigentlichen, mit Maden besetzten Fleischtheile mehr vorhanden sind, auch über dem Siebe ausgepocht. Diese Operation erfordert wenige Minuten und man wird den grössten Theil der an dem Cadaver vorhandenen Insecten nach Hause tragen und sortiren können. Auch das Entleeren der in der Erde a ufgerichteten $\mathrm{F}$ ang gläser oder Fang becher mit Fleischköder etc. soll über dem Siebe geschehen. Die darin befindlichen grösseren Käfer, wie Carabus, Silpha, Geotrupes, bleiben über dem Drahtgeflechte des Siebeinstrumentes zur Herausnahme geborgen; alle kleineren Thiere werden durch die Maschen in den Siebe sack flüchten. Die ganze Operation erfordert wenige Secunden und geschieht in gründlichster Weise. Dabei entgeht man der Gefahr, sich eine Blutvergiftung zuzuziehen. Es pflegt nämlich in die Fanggefässe oft Regenwasser zu gelangen, welches das faulende Fleisch auslaugt. Hebt man in unbesonnener Eile, wie es beim Sammeln oft vorkommt, mit von Dornen zerkratzten und aufgeschundenen Händen die in der Flüssigkeit des Bechers zappelnden Caraben etc. heraus, so kann man sehr leicht in ernste Lebensgefahr gerathen.

Durch vorstehende Winke ist aber die Anwendung des Siebes noch lange nicht erschöpft. Man findet dafür an Orten eine praktische Verwendung, wo es scheinbar nichts einzusieben gibt. Ein solcher Fall tritt an den Schneefel dern ho her Berge ein. Am hohen Pietrocza, an der Grenze NordSiebenbürgens, befinden sich in der Nähe der Schneefelder grosse Flächen, welche sich von der Ebene als Grasflächen

"Wiener Entomologische Zeitung" V. Heft 2. (20. Februar 1886.) 
ausnehmen, in der That aber aus vereinzelten Grasbüscheln bestehen, deren Wurzeln eine $10 \mathrm{~cm}$ hohe, zähe, fast undurchdringliche, dicht geschlossene Masse bilden, aus der ein Insect selbst mit dem Siebe schwer zu erlangen ist, weil diese zähe Wurzelmasse auch dem grossen Handbeile Widerstand leistete. Trotzdem gelang es mir, in diesem Wurzelwerke das Zugegensein des Niphetodes Redtenbacheri zu constatiren. Besser bewährte sich das Sieb auf dem hohen Negoi, in den transsylvanischen Alpen, wo ich Graswurzeln über's Sieb einzupfte, zwischen denen sich mehrere kostbare, zum Theile neue Coleopteren in reichlicher Anzahl vorfanden. Weit vortheilhafter ist es aber, an Schneerändern hoher Berge, Theile der vom Schneewasser durchtränkten Rasenstücke auszustechen, in Säcke verladen und entweder durch Menschen- oder Thierkräfte in's Standquartier schaffen zu lassen. Die Rasenstücke werden sodann zerkleinert und soweit trocknen gelassen, damit man zu ihrem Durchsieben oder aber direct zur Zerbröckelung auf dem Arbeitstische schreiten kann. Ich kam nur zweimal in die Lage, diese Sammelmethode anzuwenden; das erste Mal auf der Czerna-Hora der Karpathen, das andere Mal auf VelesPlanina bei Mostar. In beiden Fällen war das Resultat ausserordentlich ergiebig, obgleich die Rasen auf der Czerna-Hora bei Regenwetter ausgestochen wurden. In diesen Rasenstücken befand sich: Trechus Dejeani, Leptusa piceata, Homalota infirma Weise n. sp., carpathica, Niphetodes Redtenbacheri, Scleropterus Reitteri Weise n. sp. In den Graspolstern der Veles-Planina in der Herzegowina fand sich vor: Molops simplex, Parrayssi, Leptusa difficilis Epph. n. sp. Ocyusa nigrata, Arpedium macrocephalum zahlreich, Stomodes convexicollis Mill. n. sp. häufig und in Menge Scymnus suturalis etc.

Ein ganz unentbehrliches Sammelinstrument ist das Sieb a $\mathrm{u}$ s sumpigem Terrain. Zwei bis drei Excursionen an die Sümpfe der Narenta genügten, um viele Tausende von geschätzten Kleinkäfern heimtragen zu können. Auch an diesen Localitäten muss man nach einem gewissen System vorgehen. Vorerst siebt man das Gemülle und das Laub, oft das vorhandene Stroh oder das aufgestappelte, faulende Rohrwerk ein und man wird meist über die Massen Käfer staunen, welche diese vegetabilischen Ueberreste beherbergen. Die Bryaxis, Euconnus, Pelochares und andere Arten sind oft in unglaub- 
lichen Mengen vorhanden; die Tychus und die Boeocera finden sich zwischen den faulenden Rohrüberresten. Die zahllosen Bembidien, Staphyliniden und Anthiciden, die beim Aussuchen des Gesiebes in hastiger Geschwindigkeit davoneilen, können uns schier die Arbeit verleiden. Die schlammigen Sumpfränder sollen nach den grossen Compsochilus-Arten mit den Händen geknetet werden; diese Thiere kommen auf andere Weise schwer zum Vorschein. Die kleinen Compsochilus finden sich im Geniste der Sumpfränder oder am Schlamme zwischen den dichten Sumpfgräsern. So fand ich den Comps. Rosti auf Zante; ich entfernte die obere Schlammschichte und legte sie in's Sieb; ein grosser Theil der Käfer flüchtete in den Siebesack, während sich andere Stücke in den Schlamm tiefer einzugraben suchten. Grosse Grasbüschel, welche oftmals inse!förmig aus den Sümpfen vorragen, müssen systematisch ausgebeutet werden, da sie ein Heer von Insecten zwischen ihren Blättern und Wurzeln beherbergen. Solche Grasbüsche werden sammt den Wurzeln ausgegraben und von der Erde über dem dichten Siebe entblösst. Sodann siebt man so viel von der vorhandenen fetten Humuserde ein, als sie in Menge Käfer aufweist. Anstatt dieser Grasbüsche des Narentathales fand ich an den Sümpfen der jonischen Inseln grosse Distelbüsche, unter deren Schutz die ganze Sumpffauna Zuflucht gefunden hatte. Um zu den Siebethieren zu gelangen, musste erst der Distelbusch mit einem Beile entblättert werden; sodann wurden die alten faulenden Blattüberreste, meistens unter arger Belästigung der Hände, sammt der oberen Erdschichte in's Sieb geschafft. Von der Arten- und Individuenzahl, welche auf diese Weise in das Sieb gelangt, kann sich der Nordländer kaum einen Begriff machen, da in unseren Ländern eine ähnliche allgemeine und permanente Massenzufluchtsstätte von Coleopteren nicht vorzukommen scheint.*)

Ein sehr ergiebiges Sieberesultat lieferten auf den jonischen Inseln die überall zahlreich vorhandenen, faulenden oder verfaulten und bereits ausgedörrten Opuntia-Blätter. In dem Safte der faulenden Blätter lebt in grosser Menge das Agathidium opuntiae; in den trockenen: Euthia formicetorum,

*) Man vergleiche meine Resultate einer coleopterologischen SammelCampagne während der Monate Februar bis April 1883 auf den jonischen Inseln. Deutsch. Ent. Zeitschr. 1884, pag. 101. 
Atomarien, Monotomen, Stenosis-Arten, Dapsa opuntiae (selten) und andere. Die Blätter werden der Länge nach getheilt, damit das Innere blossgelegt erscheint und sodann durch heftige Schläge über dem Siebe ausgebeutet.

Bei Grotten-Excursionen vergesse man ebenfalls nicht das Sieb mitzunehmen. Kann man auch nicht der im schlammigen Kothe unter Steinen lebenden Sphodrus und Anophthalmus mit demselben habhaft werden, so lassen sich aber die leicht ïbersehbaren Bathyscien, welche auf fettem Humus unter Steinen sich aufzuhalten plegen, um so leichter in raschester Weise in das Sieb einraffen. Es genügt oft für diesen Zweck ein Reservesäckchen zu verwenden.

Cryptophagus-, Atomaria-, Corticaria- und verschiedene Staphylinen-Arten findet man im Sommer in grosser Menge, wenn man das schimmelnde Bansenstroh der leer. stehenden Scheunen aussiebt. Ein einziger Siebeversuch zu günstiger Zeit an passender, feuchter Localität kann grosse Mengen dieser Thierchen zu Tage fördern. Besonders hält sich an solchen Orten Cryptophagus Milleri auf, den ich bei meinem ersten Siebeversuche vor 10 Jahren in Mähren entdeckte und dabei von dieser Art allein an ca. 500 Exemplare erbeutete.

Bevor ich diesen meinen Freunden gewidmeten Artikel schliesse, möchte ich noch auf verschiedene, den Sammelerfolg mit dem Siebe betreffende Umstände aufmerksam machen, die mir noch wichtig genug scheinen, hier einen Platz zu finden. Wie ich schon früher sagte, ist die Güte der Sammellocalität von dem Feuchtigkeitsgrade abhängig, welchen sie besitzt. Eine und dieselbe Gegend kann demnach aus diesen Gründen in den verschiedenen Jahren verschieden ergiebig sein. Obgleich man das Sieb das ganze Jahr über mit Vortheil vielfältig verwenden kann, so ist das eigentliche und ergiebigste Aussieben von feuchtem Laube hauptsächlich auf das Frühjahr beschränkt, im Sommer ist es ganz unergiebig, im Spätherbste wieder lohnender. Nur das karpathische Waldgebirge in Nordungarn und dem nördlichen Theile von Ostsiebenbürgen (vielleicht auch das Glatzer und Riesengebirge), bietet auch im Hoch. sommer dem Siebenden reichliche Ausbeute, was sich von dem grossen Feuchtigkeitsgehalte dieses ganz bewaldeten Gebirgszug'es erklären lässt. Bei meinem ersten Besuche der Capella in Croatien ergab sich im Mai in ihren Trichtern durch das 
Sieb eine sehr reiche Fauna; zwei Jahre später fand ich an denselben Localitäten im Juni Alles ausgestorben.

Nicht immer entspricht eine scheinbar gute Localität den Erwartungen, welche man in sie setzt. So boten die schönen Eichenauen auf der Insel Veglia, trotzdem sie viel faulendes Laub in den erwünschtesten Lagen bargen, oder die blühenden, jungen Wälder der Insel Lesina, kaum ein nennenswerthes Insect, während die unscheinbare Erde unter einzelnen daselbst im freien Felde stehenden Carduus-Büschen Tychus rufus, Anthiciden etc. dem Siebe lieferte.

Im Süden Europas, sowie in den subtropischen Gegenden ist es nicht immer so leicht, die Localitäten zu finden, wo man mit Erfolg sieben kann. Hat man das Sieb in unseren waldreichen Ländern als Sammelinstrument zu schätzen gelernt und unternimmt zum ersten Male eine Reise nach dem Süden, so wird man meist von dem ersten Erfolge enttäuscht sein. Man muss in diesem Falle mit Geduld die verschiedenen Umstände ausnützen, die sich nach einander darbieten. Man frägt vor Allem nach Wäldern, wo man sieben könnte, und sieht sie oft von der Ferne einladend uns entgegenwinken; ist man aber zu ihnen angelangt, so finden wir meist lichte Pinienwälder auf verdorrtem Boden, welche kaum Schutz gegen die heisse Sonne gewähren. An solchen Localitäten muss man den einzelnen eingesprengten Laubbüschen, die gewöhnlich aus Lorbeer-, Karoben- und Rosensträuchern bestehen und deren Zutritt fast immer stachelige Schlingpflanzen verwehren, nachgehen. Ein kleines Handbeil, mit dem man sich den Zutritt hiezu erzwingen kann, leistet dabei unschätzbare Dienste. Auf dem schattigen Grunde im Innern dieser Büsche wird man fast immer auf mehr oder minder hoher und feuchter Humuslage das gesuchte Laub zum Einsieben vorfinden, welches besonders an Pselaphiden, kleinen Rüsslern etc. reich zu sein pflegt. Dichte Dornenhecken an altem Gemäuer oder an Gartenzäunen und an Felderrainen sind im Süden ähnliche Sammelstellen. Auch einzelne Büsche an schattigen Felswänden können mit Erfolg aufgesucht werden. Im Süden muss man meist an eigentliche Wälder in unserem Sinne verzichten, es ersetzen sie daselbst die häufig ausgedehnten Flächen mit niederem, oft aber dichtem, schattigen Buschwerk, das meist aus immergrünen Sträuchern besteht. Im Winter ist das Einsieben des abgefallenen Laubes 
der Oelbaumplantagen manchmal lohnend, in vorgerückterer Jahreszeit gar nicht. Bessere Siebestellen bietet der Süden meist auf grösseren Bergplateaus und Bergeshängen, die oftmals unseren Sammelstellen sehr ähnlich werden.

Von grosser Wichtigkeit ist es, bei grösseren Excursionen die Zeit gut und angenehm auszunützen, um bei eintretendem, längeren Regenwetter nicht zur Unthätigkeit gezwungen zu sein. Das geschieht einfach dadurch, dass man gleich eine grössere Anzahl von Reservesäckchen mit Gesiebe einzufüllen und sich einen Gesiebevorrath zu erhalten sucht, der nach und nach ergänzt wird. Es ist selbstverständlich, lass man stets das zuerst eingetragene Gesiebe zuerst aussucht und so vorgeht, dass keines über 8-10 Tage undurchsucht bleibt. Ist das Wetter günstig, so suche man die Austrocknung des Gesiebes zu verzögern, bei schlechtem Wetter hingegen durch Vertheilung in grössere Reservesäcke oder auf andere Weise, durch Wärme etc. zu beschleunigen.

Hiermit glaube ich das Wichtigste über die Anwendung des Insectensiebes gesagt zu haben, und zwar that ich dies hauptsächlich vom Standpunkte des Coleopterologen. Neben den Coleopteren habe ich jedoch stets auch die meisten Insecten aus anderen Ordnungen von meinen grösseren Excursionen mit. genommen, welche sich in Gesellschaft der Coleopteren in dem Gesiebe vorfanden. Ich habe auf diese Weise eine beträchtliche Zahl neuer Hemipteren, meist subterrane Arten, die zum grössten Theile von Herrn v. Horvath und Dr. O. M. Reuter beschrieben wurden, zahlreiche Microhymenopteren, Arachniden und Myriopoden, welche insgesammt noch nicht wissenschaftlich verwerthet wurden, erbeutet. Nebst diesen Insecten habe ich aber auch eine Anzahl kleiner Conchylien eingesiebt und ausgelesen, welche zum Theile sehr kostbaren, zum Theile neuen Arten angehörten; Herr Dr. O. Boettger in Frankfurt hat dieselben successive beschrieben.

Wenn die Entomologen durch vorstehenden Artikel sich veranlasst finden sollten, das Insectensieb ausgiebiger zu gebrauchen, es in praktischer Weise benützen und schätzen zu lernen, so habe ich dadurch meinen $Z$ weck erreicht. Ich wünsche Allen eine gute und reiche Beute! 


\section{$2 \mathrm{BHL}$ Biodiversity Heritage Library}

Reitter, Edmund. 1886. "Das Insectensieb, dessen Bedeutung beim Fange von Insecten, insbesondere Coleopteren und dessen Anwendung. Schluss." Wiener entomologische Zeitung 5, 45-56.

https://doi.org/10.5962/bhl.part.20553.

View This Item Online: https://www.biodiversitylibrary.org/item/43771

DOI: https://doi.org/10.5962/bhl.part.20553

Permalink: https://www.biodiversitylibrary.org/partpdf/20553

\section{Holding Institution}

Smithsonian Libraries

\section{Sponsored by}

Smithsonian

\section{Copyright \& Reuse}

Copyright Status: NOT_IN_COPYRIGHT

This document was created from content at the Biodiversity Heritage Library, the world's largest open access digital library for biodiversity literature and archives. Visit BHL at https://www.biodiversitylibrary.org. 